\title{
Isolated succinate-CoQ reductase deficiency
}

INSERM

\section{Source}

INSERM. (1999). Orphanet: an online rare disease and orphan drug data base. Isolated succinate-COQ reductase deficiency. ORPHA:3208

A rare, mitochondrial oxidative phosphorylation disorder characterized by a highly variable phenotype. The severe, multisystemic disease involves brain, heart, muscles, liver, kidneys, and eyes and results in death in infancy. Mildly affected individuals have only isolated cardiac or muscle involvement in the adulthood. Histochemical and biochemical analysis reveals a global reduction of succinate dehydrogenase activity. 